\title{
Best practice with regard to confirmation of nasogastric tube placement
}

\author{
M. A. Thomson ${ }^{1}$, L. Lawson ${ }^{1}$ and M. Shaw ${ }^{2}$ \\ ${ }^{1}$ Department of Nutrition and Dietetics, Lynebank Hospital and ${ }^{2}$ Department of Surgery, Queen Margaret Hospital, \\ Dunfermline, Fife KY11 4UW, UK
}

There have been an increased number of significant clinical incidents at both local and national level with regard to misplaced nasogastric (NG) feeding tubes, many with adverse outcomes. Between 2002 and 2004 at least eleven patients died as a result of misplaced tubes and a further thirteen incidents involving NG feeding tubes were reported to the National Patient Safety Alert's (NPSA) national reporting body.

NG feeding tubes are used to provide liquid nutrition, fluid and medication to patients who require short-term nutritional support. NG feeding is common practice and thousands of tubes are placed daily within wards and community settings without incidence. There is however a small risk that the tube can be placed or become displaced into the lungs during insertion, or move out of the stomach at a later stage $^{(1)}$.

A number of studies have shown that conventional methods such as auscultation, checking with litmus paper and visual inspection of the aspirate are grossly inaccurate. Furthermore there is a great deal of conflicting advice regarding the need for radiology to confirm the position of tubes. Whilst this method remains necessary for a small and select group of patients, it is not required for the vast majority of patients receiving enteral feeding. The confusion as to the most effective evidence based method of checking tube position resulted in varied clinical practice across health regions.

The NPSA ${ }^{(2)}$ issued guidance to staff and patients as to the correct methods that should be used to confirm the position of NG feeding tubes.

The aim of this project was to produce a nursing and midwifery clinical guidance document in a poster format for the confirmation of a NG tube in adults using $\mathrm{pH}$ indicator strips. The anticipated outcomes were that there would be a reduced clinical risk and improved patient safety in patients fed via an NG feeding tube by adopting the correct method(s) to confirm tube placement. This outcome would be measurable by reviewing number of reported incidents on Datix (NHS Fife incident/near miss reporting system).

In addition it was anticipated that there would also be a reduced number of inappropriate requests for X-ray to confirm tube position as the guidance document gave instructions and strategies to adopt if there was any difficulty obtaining aspirate.

The clinical guidance document was widely distributed throughout NHS Fife to all professions with an interest or involvement in NG feeding for comments. The final document encompassing the changes was ratified and signed off by the NHS Fife clinical effectiveness team in January 2007.

In essence, the authors used the main brief of the NPSA together with the key recommendations included within the alert to produce a poster flow guide in a pictorial manner for NHS Fife staff. Litmus paper was removed from all the wards and departments and the Area Supplies Centre was instructed only to supply $\mathrm{pH}$ indicator strips (Merck 2-9) to wards and departments. The poster had three main sections; what to do if $\mathrm{pH} \leq 5.5$, what to do if $\mathrm{pH}>5.5$ and steps to take if no aspirate was able to be obtained. The pictorial nature of the poster meant that where there was difficulties obtaining aspirate or, where the aspirate was $>5.5$, it was easier for staff to visualise what the cause/problem might be and to be able to react accordingly. The poster also gave advice for staff regarding pH changes in patients on protein pump inhibitors and actions to take in these cases to confirm position.

The poster was displayed in all wards and clinical areas where NG feeding could possibly take place. The NPSA alert/poster was incorporated into the monthly enteral tube feeding training sessions to ensure as many staff as possible became aware or updated regarding risks and best practice. Nursing staff report that they feel significantly more confident in confirming the position of Nasogastric feeding tubes when using the poster.

1. Tait J (2001) Complete Nutr 1, 27-29.

2. NHS National Patient Safety Agency (2005) Patient safety alert 05. http://www.npsa.nhs.uk/patientsafety/alerts-and-directives/alerts/nasogastricfeeding-tubes/ 